\title{
Montreal Cognitive Assessment Visuospatial/Executive Test
}

National Cancer Institute

\section{Source}

National Cancer Institute. Montreal Cognitive Assessment Visuospatial/Executive Test. NCI Thesaurus. Code C162709.

These two abilities are assessed through the Trails B Test, which requires an individual to draw a line to correctly sequence alternating digits and numbers (1-A, 2-B, etc.) and through a task which requires them to draw a copy of a cube shape. 